\title{
Morphology and clinical implication of the extra-head of biceps brachii muscle
}

\author{
Ashraf Y. Nasr ${ }^{1}$ 2, Adel M. Hussein ${ }^{2}$ \\ ${ }^{1}$ Anatomy Department, Faculty of Medicine, Zagazig University, Zagazig, Egypt \\ ${ }^{2}$ Anatomy Department, Faculty of Medicine, King Abdul-Aziz University, Jeddah, Kingdom of Saudi Arabia
}

[Received 5 May 2013; Accepted 23 June 2013]

\begin{abstract}
The biceps brachii muscle is present in the anterior aspect of the arm. Its morphological variations have great clinical significance for surgeons, orthopaedic surgeons, anaesthetists, neurologists and anatomists. This study aimed to describe the incidence and morphology of the extra-heads of the biceps brachii muscle. Hundred upper limbs of 50 adult human cadavers (30 men and 20 women) were used in this study after the approval of the medical ethical committee. These cadavers were obtained from the Anatomy Department, Faculty of Medicine, King Abdul-Aziz University. The incidence of anatomical variations of biceps muscle was equal in both male and female cadavers (10\%) with predominance of the left side (7\%). The 3-headed biceps brachii muscle was noticed in 7\% (4\% male and 3\% female), while the 4-headed biceps was seen in 2 (2\%) left limbs, 1 male and 1 female. The third head of the biceps muscle arose from the anteromedial aspect of humerus, between the coracobrachialis insertion and the brachialis origin, in 6\% and from middle of the medial border of humerus in $3 \%$. While the fourth head originated from the articular capsule of shoulder joint in 1 (1\%) limb and from the coracoid process of scapula in the other limb. The biceps common tendon of insertion received the supernumerary heads in $7 \%$ of the limbs. However, the extra-head fused with the long head in 2 (2\%) limbs and united with the short head in 1 (1\%) limb. The mean of the third head length was $118.8 \pm 10.9$ in all limbs, where it was $121.8 \pm 12.3$ in male and $113.5 \pm 8.1$ in female cadavers. The third head length/arm length ratio was $38.4 \pm 2.6$ in all, $38.3 \pm 3.4$ in male and $38.8 \pm 1.8$ in female cadavers. The length of the extra-head was extremely significant with those of the corresponding limb in all, male and female cadavers ( $p<0.0001)$. Knowledge of the morphological variations of biceps muscle provides better pre-operative evaluation, safe surgical intervention within the arm and better postoperative outcomes. (Folia Morphol 2013; 72, 4: 349-356)
\end{abstract}

Key words: biceps brachii muscle, extra-head, morphology, variations

\section{INTRODUCTION}

The biceps muscle is present as one of the anterior compartment muscles of the arm. It has short and long heads. The long head of the biceps arises from supragle- noid tubercle of scapula. The short head of the biceps originates from coracoid process by a thick flattened tendon in common with coracobrachialis muscle. Both bellies of the biceps muscle unite in a flat tendon which 
is inserted in the tuberosity of the radius and the bicipital aponeurosis. This bicipital aponeurosis is attached to the medial border of the tendon and fused with the deep fascia of the forearm and the posterior border of ulna. This form of insertion provides an efficient supination of the forearm [23].

The original variations of biceps brachii are not rare. These variations may be presented as a group of fascicles that arise from coracoid process, tendon of pectoralis major or as an extra-head arising from the humeral head, bicipital groove, capsule of the shoulder joint, the shaft of humerus or from coracoid process $[8,18,19]$. From 1 to 7 extra-heads of biceps were reported [12, 24]. These variations had been found in about $9-22 \%$ of cadavers [5]. In addition, insertional variations of the biceps muscle were reported as well [15].

The humeral head of biceps is a thin flattened fascicle that lies below the biceps muscle. It arises from the shaft of humerus, between brachialis and coracobrachialis muscles, or between the brachialis muscle and the radial groove. Sometimes, it arises from anterior surface of the brachialis muscle [18].

There is a wide range of variations of extra heads of biceps brachii according to race. The incidence of occurrence of these variations is $37.5 \%$ in Colombians [18], 21.5\% in South Africans blacks, $8.3 \%$ among South African whites [3], 18\% in Japanese [7], 15\% in Turkish [16], 7.1\% in Indians [17].

The aim of the present study is to describe the morphological features and the incidence of extra-heads of the biceps brachii. The knowledge of these topics has a great clinical significance for surgeons, anaesthetics, neurologists and orthopaedic surgeons during surgical interventions within the arm and also for academic study.

\section{MATERIALS AND METHODS}

Hundred upper limbs of 50 human adult cadavers (30 men and 20 women) were used in this study. The cadavers were obtained from the Anatomy Department, Faculty of Medicine, King Abdul-Aziz University, Jeddah, Kingdom of Saudi Arabia after approval of the medical ethical committee. A vertical incision was made on the anterior aspect of the arm from the level of acromion to the level below the elbow crease by $5 \mathrm{~cm}$. Another 2 transverse incisions were made perpendicular to the upper and lower ends of this vertical incision. Skin and subcutaneous fascia were removed to expose the biceps brachii muscle. The origin, insertion, presence of extra-head, pattern of innervation of each biceps muscle were carefully observed, recorded and photographed. The data were tabulated in an excel sheet regarding side and sex of the limb. The incidence of any variant or anomaly of the biceps muscle were recorded and calculated in number and percentage. The length of the supernumerary heads and the arm was measured by using a Verneir caliper of accuracy $0.01 \mathrm{~mm}$. The mean and standard deviation (SD) of the length of supernumerary heads and arm as well as supernumerary head length/arm length ratio were calculated through statistical analysis using SPSS software version 16 . Student $t$-test was used to determine the significance of the extra-head length to the arm length. The value was considered significant at $p$ value $<0.05$.

\section{RESULTS}

The morphology and morphometric study of the extra heads of the biceps muscle were reported (Table 1). The morphological variations of the biceps muscle were seen in 10 out of $100(10 \%)$ cadavers. Its original variations were seen in $9(9 \%)$ limbs, while the abnormal insertion was noticed in 1 right male limb only. These variations were equally distributed in both male and female cadavers, $10 \%$ each. The third head of the biceps muscle arose from anteromedial aspect of humerus, between the coracobrachialis insertion and the origin of brachialis muscle, in $60 \%$ of the variations (Fig. 1), while the middle of the medial border of humerus gave origin to the third head of the biceps muscle in $30 \%$ of the variants (Fig. 2). These extra-heads were inserted into the common tendon of biceps muscle in $70 \%$ of the variant cases (Fig.1). The long head of biceps received the extra-heads in $2(20 \%)$ cases (Figs. 2, 3) and the supernumerary heads united with short head of biceps muscle in $1(10 \%)$ limb only (Fig. 4). The fourth head of the biceps originated from the capsule of the shoulder joint in a male left arm (Fig. 3) and from the coracoid process in a female left arm (Fig. 4). An abnormal variation of the biceps insertion was seen in a right arm of male cadaver where the common tendon gave a slip into the brachialis muscle proximal to its insertion in the posterior part of radial tuberosity. On the contrary, the brachialis muscle gave a muscular band to the under surface of the common tendon of the biceps muscle (Fig. 5).

The incidence of morphological variations of biceps muscle was equally distributed in both male and female cadavers. Regarding the limb side, a left side predominance of 7 out of 10 variants was observed in both male and female cadavers. These variations 
Table 1. Morphology and morphometry of the supernumerary biceps heads

\begin{tabular}{|c|c|c|c|c|c|c|c|}
\hline Sex & $\begin{array}{l}\text { Variant } \\
\text { biceps }\end{array}$ & $\begin{array}{l}\text { Limb } \\
\text { side }\end{array}$ & $\begin{array}{l}\text { Origin of the extra-head } \\
\text { of biceps }\end{array}$ & $\begin{array}{l}\text { Insertion of } \\
\text { extra-head }\end{array}$ & Variant length (VL) & Arm length (AL) & $\mathrm{VL} / \mathrm{AL} \times 100$ (ratio) \\
\hline \multirow[t]{7}{*}{ Male } & Three-headed & Left & AMS & Common & 138 & 325 & $42.46 \%$ \\
\hline & & & Med. border & LH & 105 & 315 & $33.3 \%$ \\
\hline & & & AMS & Common & 118 & 318 & $37.11 \%$ \\
\hline & & & Med. border & Common & 120 & 310 & $38.7 \%$ \\
\hline & Four-headed & Left & Joint capsule & Common & 280 & 320 & $87.5 \%$ \\
\hline & & & AMS & 내 & 128 & & $40 \%$ \\
\hline & Insertion & Right & Common tendon & Brachialis & 78 & 330 & $23.6 \%$ \\
\hline \multirow[t]{5}{*}{ Female } & Three-headed & Left & Med. border & Common & 110 & 300 & $36.7 \%$ \\
\hline & & & AMS & Common & 124 & 310 & $40 \%$ \\
\hline & & Right & AMS & Common & 115 & 290 & $39.66 \%$ \\
\hline & Four-headed & Left & Coracoid process & CH & 275 & 295 & $93.2 \%$ \\
\hline & & & AMS & DII & 105 & & $35.6 \%$ \\
\hline
\end{tabular}

AMS — anteromedial surface of humerus; LH — long head; SH — short head

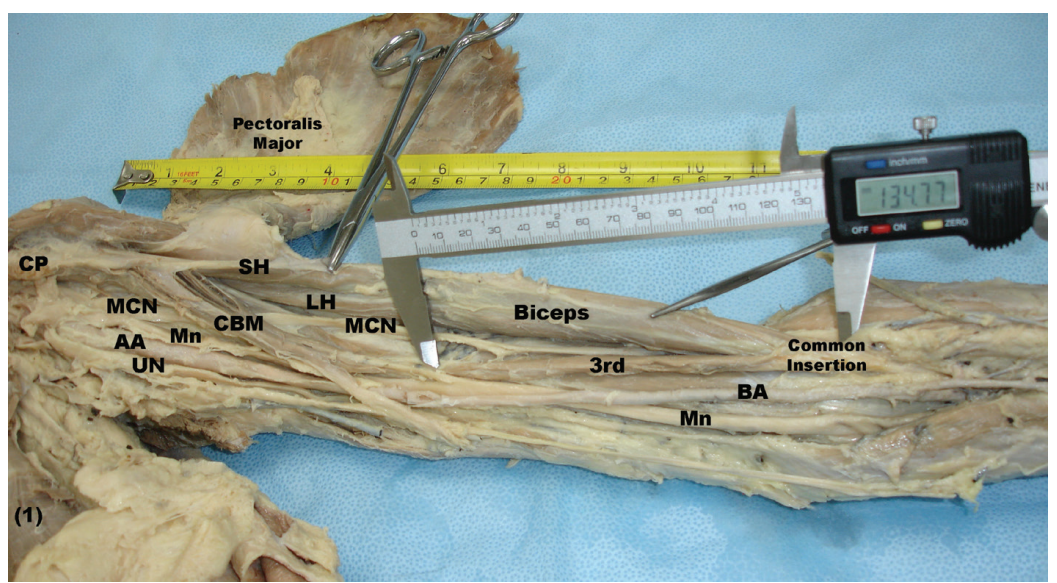

Figure 1. Left female upper limb showing the third head of biceps $\left(3^{\text {rd }}\right)$ originating from the anteromedial aspect of the humerus distal to the coracobrachialis muscle (CBM) insertion and inserts into the common bicipital tendon. The short head (SH) and CBM originate from the coracoid process (CP); $\mathrm{MCN}$ — musculocutaneous nerve; $\mathrm{AA}$ — axillary artery; $\mathrm{Mn}$ — median nerve; Un — ulnar nerve; BA — brachial artery.

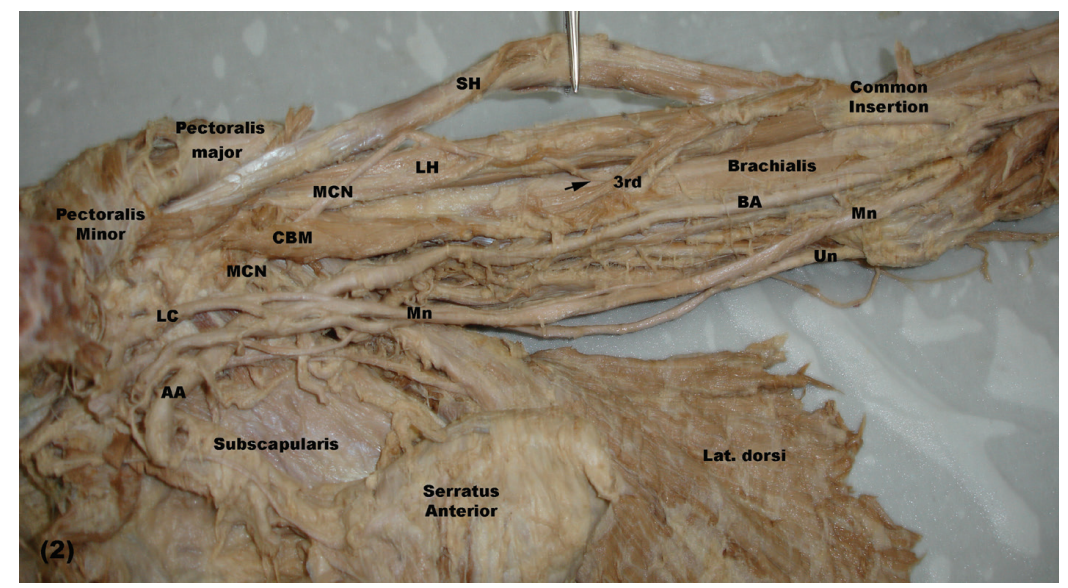

Figure 2. Left female upper limb showing the third head of biceps $\left(3^{\text {rd }}\right)$ originating from the middle of medial border of the humerus and inserting into long head (LH). The musculocutaneous nerve (MCN) pierces the coracobrachialis muscle (CBM) to supply brachialis muscle and short head (SH), $\mathrm{LH}$ and third head $\left(3^{\text {rd }}\right)$ of the biceps muscle (arrow); LC — lateral cord; $\mathrm{Mn}$ — median nerve; $\mathrm{Un}$ — ulnar nerve; AA — axillary artery; BA — brachial artery. 


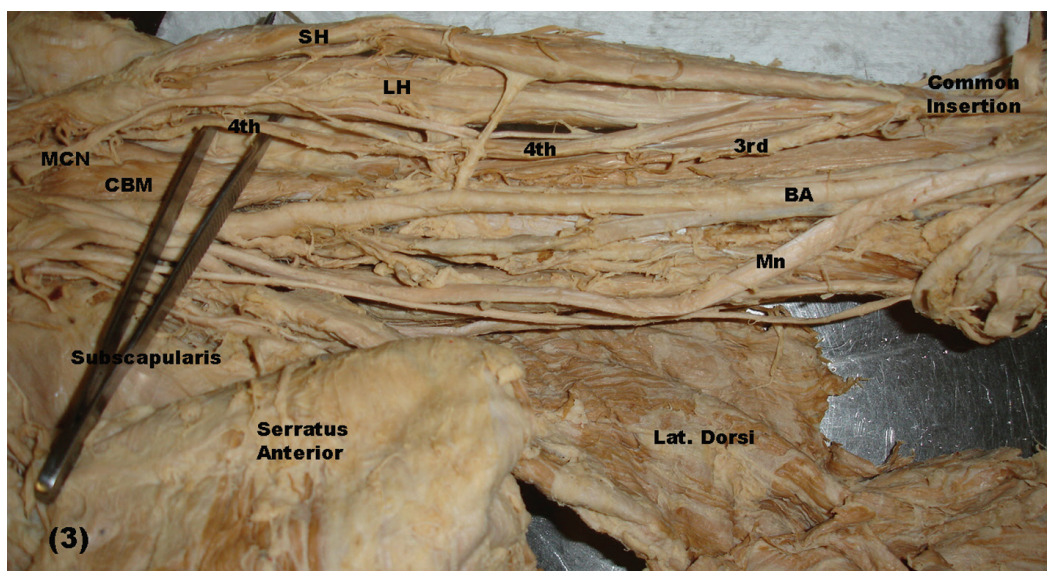

Figure 3. Left male upper limb showing four-head biceps muscle. The third head $\left(3^{\text {rd }}\right)$ originates from anteromedial surface of humerus between coracobrachialis muscle (CBM) insertion and brachialis muscle origin. It inserts into the common tendon of the biceps muscle, while the fourth head $\left(4^{\text {th }}\right)$ originates from the articular capsule of shoulder joint and inserts into the long head (LH). The musculocutaneous nerve (MCN) supplies all heads of biceps muscle; SH — short head; $\mathrm{Mn}$ — median nerve; BA — brachial artery.

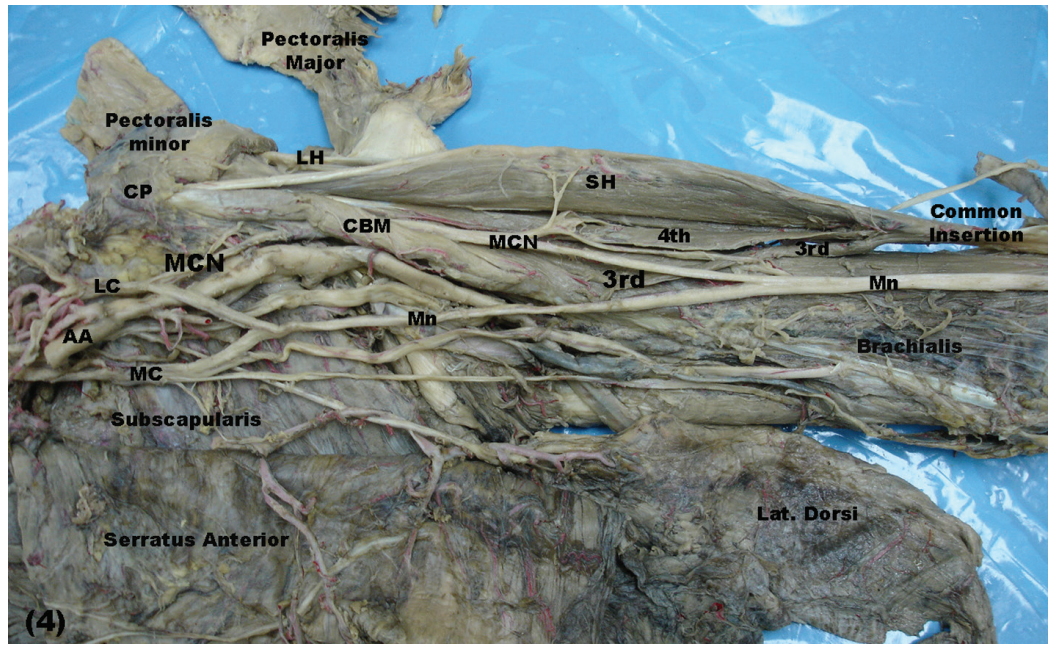

Figure 4. Left female upper limb showing the four-headed biceps muscle. The third head $\left(3^{\text {rd }}\right)$ originates from the anteromedial surface of humerus between coracobrachialis insertion (CBM) and brachialis muscle origin. It inserts into the common tendon of biceps. The fourth head $\left(4^{\text {th }}\right)$ originates from coracoid process (CP) and inserts into the under surface of the short head (SH). The musculocutaneous nerve (MCN) gives an abnormal communicating branch to the median nerve (Mn) proximal to the muscular branches of biceps' heads and brachialis muscles; LH — long head of biceps; LC — lateral cord of brachial plexus; MC — medial cord of brachial plexus; AA — axillary artery.

were seen in both origin and insertion of the muscle. Nine out of the ten variants were of the original type. The incidence of the 3-headed biceps brachii muscle was seen in $7 \%$ of the total limbs: 4 out of $60(6.7 \%)$ male and 3 out of 40 (7.5\%) female limbs. Moreover, the 4-headed biceps muscle was noticed in $2(2 \%)$ left limbs: $1(1.7 \%)$ male and 1 (2.5\%) female. However, an insertional variation of the biceps muscle was noticed in only $1(1 \%)$ right male limb (Table 2 ).

The measurements of the biceps muscle extraheads are reported in Table 3 . The mean length of all extra-heads of the biceps muscle measured was
$118.1 \pm 10.9 \mathrm{~mm}$. The ratio of that mean in comparison with those of the arm length was $34.4 \pm 2.6$. In male cadavers, the mean length of the extra heads of the biceps muscle was $121.8 \pm 12.3 \mathrm{~mm}$ and its rational length was $38.3 \pm 1.5$ of the arm length. While in female cadavers, the mean length of the third head was $113.5 \pm 8.1 \mathrm{~mm}$ and it represented $38.8 \pm 1.8$ of arm length $(p<0.0001)$.

All the extra heads of the biceps muscle were supplied by the musculocutaneous nerve through separate branch or from the motor branches of short and/or long heads of the biceps muscles (Figs. 1-4). 


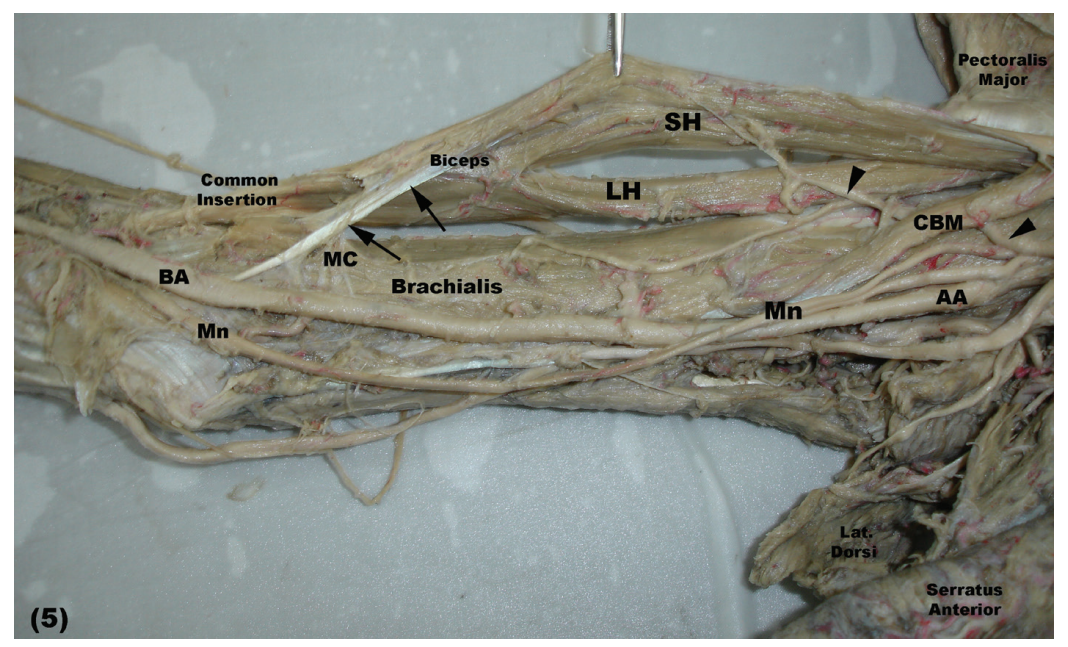

Figure 5. Right male upper limb showing an abnormal muscular communication between the biceps muscle (arrow) and the brachialis muscle. The brachialis muscle gives a muscular band (MC) to the under surface of the abnormal bicipital sling; SH — short head; LH — long head of biceps; CBM — coracobrachialis muscle; AA — axillary artery; $\mathrm{Mn}$ - median nerve; BA — brachial artery; UN — ulnar nerve; arrow head — musculocutaneous nerve.

Table 2. Incidence of biceps variations

\begin{tabular}{|c|c|c|c|c|c|c|c|c|}
\hline & \multicolumn{4}{|c|}{ Origin variations } & \multicolumn{4}{|c|}{ Insertion variations } \\
\hline & \multicolumn{2}{|c|}{ Male } & \multicolumn{2}{|c|}{ Female } & \multicolumn{2}{|c|}{ Male } & \multicolumn{2}{|c|}{ Female } \\
\hline & Right & Left & Right & Left & Right & Left & Right & Left \\
\hline Number (\%) & $1(3.3 \%)$ & $4(13.3 \%)$ & $1(5 \%)$ & $3(15 \%)$ & $1(3.3 \%)$ & 0 & 0 & 0 \\
\hline \multirow[t]{2}{*}{ Total (N/\%) } & & & & & & & & \\
\hline & \multicolumn{4}{|c|}{$9(9 \%)$} & \multicolumn{4}{|c|}{$1 \%$} \\
\hline
\end{tabular}

Table 3. Length of third head of biceps and third head length/arm length ratio

\begin{tabular}{lcccccccc}
\hline & N & Mean & SEM & SD & Minimum & Maximum & P value & t value \\
\hline Length: all & 9 & 118.1 & 3.6 & 10.9 & 105.0 & 138.0 & $<0.0001^{\text {a }}$ & 21.333 \\
Ratio: all & 9 & 38.4 & 0.87 & 2.61 & 33.3 & 42.5 & NS & - \\
Length: male & 5 & 121.8 & 5.48 & 12.26 & 105.0 & 138.0 & $<0.0001^{\text {b }}$ & 14.669 \\
Ratio: male & 5 & 38.3 & 1.53 & 3.42 & 33.3 & 42.5 & NS & - \\
Length: female & 4 & 113.5 & 4.05 & 8.10 & 105.0 & 124.0 & $<0.0001^{c}$ & 17.996 \\
Ratio: female & 4 & 38.8 & 1.05 & 1.82 & 36.7 & 40.0 & NS & - \\
\hline
\end{tabular}

$a, b, c$ The difference between the length of the third head and the arm length in the corresponding group is significant at $p<0.0001$; NS — not significant

\section{DISCUSSION}

The number, morphology, origin, insertion, innervation and racial differences of the heads of biceps muscle had been previously studied $[1,4,8,16,17$, 20]. Occurrence of biceps third head is a common variant $[10,17]$, but the presence of 4,5 or more heads have been reported [24]. However, rare variations had been reported regarding the biceps insertion, absence of its long or short heads $[5,13]$.
Incidence of the extra heads of the biceps muscle was quite frequent, where the 3 -headed biceps brachii muscle was $10 \%$ in white Europeans, $12 \%$ in black African populations, $8 \%$ in Chinese, $18 \%$ in Japanese, $13 \%$ in European, $15 \%$ in Turkish, $20 \%$ in white Brazilian population and $9 \%$ in black Brazilian population [12, 17, 21, 23]. Moreover, in Colombian population, the incidence of the third head of the biceps muscle was 37.5\% [18]. However, Arora and Dhingra [2], Kumar 
et al. [8] had recorded that the incidence of the third head of the biceps muscle varied from $3 \%$ to $1 \%$ of the examined limbs. In comparison with the results of previous researches, the 3 -headed biceps brachii muscle was seen in $7 \%$ of the specimens in the present study.

In accordance with Cheema and Singla [4], Amar and Elezy [1], in Indian population, the present results revealed that the occurrence of the third head of biceps muscle was more on left arms of both male and female cadavers. However, researches of Rincon et al. [18] in the Colombian, Rai et al. [17] in Indian population, Poudel and Bhattarai [16] in Nepalese, Kervancioglu and Orhan [6] in Turkish foetuses, stated that, the incidence of the third head of the biceps muscle was more often seen in the right arms. Not only a unilateral third head was reported by Ozan et al. [13], Sharadkumar et al. [22], Amar and Elezy [1] but also, bilateral cases were observed by Rincon et al. [18] and Sandeep et al. [20] as well. However, no bilateral case of an extra head of the biceps muscle was reported in this study. The incidence of the extra heads of biceps muscle varies among the ethnic groups [18]. The incidence difference of such variation might be attributed to evolutionary and/or racial factors [12]. The predominance of male right side of the supernumerary head of the biceps muscle might be due to the functional adaptation in the people who do more physical exercise [18].

In this study, $70 \%$ of the third heads originated from the humerus below the insertion of the coracobrachialis muscle and above the brachialis muscle origin, while they originated from middle of the medial border of the humerus in $30 \%$ of the specimens. In agreement with the present results, Cheema and Singla [4], Amar and Elezy [1], Sandeep et al. [20] and Rai et al. [17] reported that in Indian population, the third head of the biceps muscle was commonly taking origin from the anteromedial surface of humerus. Also, the third head in Turkish foetuses arose from the anteromedial aspect of the humerus [6]. However, both of the anteromedial and anterolateral surfaces of the humerus gave origin of an equal number of the supernumerary heads of biceps muscle [22]. Moreover, 3 distinct original sites of the extra-head of the biceps muscle were reported by Asvat et al. [3], but with different occurrence frequency. The later authors added that the commonest site of origin of that extra head of the biceps was the medial aspect of the humerus in common with the insertion of the coracobrachialis muscle, while the least one was a fascial origin from the medial side of deltoid muscle and the lateral side of the short head of biceps muscle. In disagreement with the present study, the third head of the biceps muscle was formed of a group of fascicles that arose from the coracoid process of scapula, the pectoralis major tendon or from the anterior aspect of humerus just below the lesser tubercle crest [21], head of humerus, the articular capsule of shoulder joint [18] or from the shaft of humerus itself $[7,8]$.

The insertion sites of the extra-heads of biceps were classified into 4 main groups, but with different occurrence rates. The highest incidence site of the extra-head insertion was the common belly of the biceps that received $73.3 \%$ of the extra-heads, while the least one was the insertion into the long head (2.7\%). The biceps tendon and the short head received the extra-head insertion in $14.7 \%$ and $9.3 \%$, respectively $[7,11]$. In agreement with a previous report, the present results revealed that $70 \%$ of the extra-heads of the biceps muscle inserted into the common belly of the biceps, while, each of long and short head received $15 \%$ of them. Moreover, the extra-heads of biceps can be inserted into the muscular belly or the aponeurosis of the biceps muscle [19]. A racial or a developmental factor might be the actual cause of the incidence difference of the insertion of the extra-head in this present study and the other previous literatures.

The origin of the extra-heads from the antromedial surface of humerus might support the opinion that the pronation of forearm occurs in any position of the shoulder joint. The presence of third head of the biceps muscle might increase the strength of flexion of the elbow [11].

Rare variations regarding the insertion of biceps have been reported and also the absence of its long or short heads [13]. In the present study a single variant of the biceps insertion was observed. In this variant, biceps muscle insertion bifurcated into medial and lateral parts. The lateral part continued forming a common tendon that was inserted in the posterior part of the radial tuberosity and also in the bicipital aponeurosis, while the medial part passed deep to the brachial artery and the median nerve and it fused with the insertion of brachialis muscle in the ulna. The presence of such variant might enhance the supination as well as flexion of the forearm [18].

Similar observations were recorded by Paval and Mathew [15] who reported that some fibres from the medial side of the biceps brachii tendon formed a separate narrow tendinous slip that was subdivided into 
lateral superficial and medial deep slips surrounding the brachial artery and median nerve. The later authors added that, this case was mimicking the situation of the median nerve compression underneath the bicipital aponeurosis and this variation might be one of the causes of pronator teres syndrome. The variants of biceps muscle insertion were also noticed, where some slips of this muscle might extend to the medial epicondyle, the medial intermuscular septum of the arm, the brachialis muscle or the pronator teres muscle [12]. The later authors added that variations had important clinical significance regarding surgical approaches.

Embryologically, the third head of the biceps muscle was considered as a part of brachialis muscle that was separated by the musculocutaneous nerve, where its lower insertion migrated from ulna to radius [18]. The presence of the third head of the biceps muscle in humans might represent the remnant of long head of the coracobrachialis of some other primates $[9,25]$. The presence of the extra heads of the biceps muscle with or without neural variation might be due to change of signal between a group of mesenchymal cells and neuronal growth factors of forearm muscles developed from the paraxial mesoderm during embryonic development $[9,23]$, or it might be caused by the presence of the circulatory factors during the time of formation of brachial plexus [11].

From the functional point of view, the extra head of the biceps brachii muscle with a humeral origin might allow flexion of the elbow joint in any position of the shoulder joint [24]. The dual origin of the third head of the biceps might contribute to supination of forearm, as the muscle origin appeared in a lateral position relative to the rotational axis of the arm. The medial brachial origin of the third head might contribute to pronation of the forearm in any position of the shoulder joint. In addition, the third head could strengthen the flexion of the elbow [25].

The 4-headed biceps muscles were rare in comparison to that of the 3-headed biceps muscles [12]. The 4-headed biceps muscle in the present study was seen in $2(2 \%)$ left arms: 1 male and 1 female. The supernumerary head of biceps muscle was commonly originated from an area between lesser tubercle and origin of brachialis muscle $[19,23]$. However, in the present study the fourth head of biceps brachii originated in 1 case from the coracoid process and from capsule of shoulder joint in the other case.

The extra head of the biceps muscle that originated from the tendon of pectoralis major, coracoid process of scapula, articular capsule of the shoulder joint, head of humerus or shaft of humerus might cause bone displacement subsequent to fracture [11].

The tip of acromion process and lateral epicondyle were used as reference points of the arm length [14]. The later authors added that, this method was the most useful and more accurate as the measurements were easy to record and simple to use clinically. Similar method was used in the present study to determine the arm length, extra-head length and the extra-head length/arm length ratio.

The present study revealed that the mean of the arm length in the male cadavers measured $121.8 \pm$ $\pm 12.3 \mathrm{~mm}$ and that of the female cadavers was $113.5 \pm 8.1 \mathrm{~mm}$. When comparing the length of the supernumerary head to that of the arm, it was $38.3 \pm$ $\pm 3.4 \%$ in male and $38.8 \pm 1.8 \%$ in female cadavers. In disagreement with those of the present study, the length of third head measured $109.9 \mathrm{~mm}$ at radial side and 90-99.3 mm from ulnar side [7]. However, the incidence of the extra-head of biceps muscle was commonly on the right male upper limbs $[1,18]$. The later authors added that there was a concomitant correlation between the incidences of the supernumerary heads of the biceps brachii muscle with the dominant hands of the population. Sharadkumar et al. [22] had reported an equal distribution of the third head in both right and left upper limbs. Similar observations were noticed in the present study, where the extra-heads of biceps were equally distributed between right and left upper limbs in both male and female cadavers. Thus, the distribution of both right- and left-handed people might be show an equal distribution in the community.

Similar to the present results, Poudel and Bhattarai [16] in Nepalese, Cheema and Singla [4], Amar and Elezy [1], Kumar et al. [8] in Indians reported that, the third head of biceps brachii was innervated mostly by a branch from musculocutaneous nerve and its arterial supply was from the brachial artery.

No clinical manifestations were recorded in most of the limbs having a supernumerary head of the biceps muscle, therefore the supernumerary head of this muscle might be misinterpreted as being soft tissue tumour. Moreover, in certain cases, the presence of such extra-head of biceps could produce a compression of median nerve or brachial artery in the arm $[12,17]$. Although, bicipital variations of biceps muscle are of great interest to the anatomists, surgeons and orthopaedic surgeons. They must be aware of such muscular variations, since unusual clinical manifestations at the upper limb may be 
produced as a result of such anatomical variations. Moreover, more attention is needed during exposure of the front arm muscles or shoulder joint, where identification and mobilisation of any extra-head of the biceps muscle is necessary for adequate exposure of the shoulder joint through a deltopectrol incision [10]. Thus, knowledge of these variations might provide better preoperative diagnosis, facilitation of the surgical intervention and good postoperative prognosis.

\section{CONCLUSIONS}

The anatomic variations of the biceps brachii muscle are very important for clinicians who perform surgical intervention in the arm. Therefore, surgeons, orthopaedic surgeons, anaesthetists and also anatomists should be aware of the incidence of the anatomical variations of the biceps muscle. Although the short and long heads of the biceps brachii muscle are not suitable enough for the coverage of defects of soft tissue in arm, the extra heads of the biceps muscle may be possible and of expandable value for any procedure done by the surgeons, orthopaedic surgeons and/or the plastic surgeons.

\section{ACKNOWLEDGEMENTS}

Great appreciations to all technical members of the Anatomy Department, Faculty of Medicine, King Abdul-Aziz University for their help during doing this work.

\section{REFERENCES}

1. Amar J, Elezy MN (2012) Study of variations in the origin of biceps brachii muscle in Kerala. Int J Sci Res, 2: 1-4.

2. Arora L, Dhingra R (2006) Unusual nerve supply of biceps from ulnar nerve and median nerve and a third head of biceps. Indian J Plastic Surg, 39: 172-174.

3. Asvat R, Candler P, Sarmiento EE (1993) High incidence of the third head of biceps brachii in South African populations. J Anat, 182: 101-104.

4. Cheema P, Singla R (2011) Lower incidence of the third head of biceps brachii in North Indian Population. J Clin Diagnostic Res, Suppl. 2, 5: 1323-1326.

5. Cucca YY, SVB McLay, T Okamoto, J Ecker (2010) The biceps brachii muscle and its distal insertion: observations of surgery and evolutionary relevance. Surg Radiol Anat, 32: 371-375.

6. Kervancioglu P, Orhan M (2011) An anatomical study on the three-headed biceps brachii in human foetuses, and clinical relevance. Folia Morphol, 70: 116-120.

7. Kosugi K, Shibata S, Yamashita H (1992) Supernumerary head of biceps brachii and branching pattern of the musculocutaneous nerve in Japanese. Surg Radiol Anat, 14: 175-185.
8. Kumar H, Das S, Rath G (2008) An anatomical insight to the third head of biceps brachii muscle. Bratisl Lek Listy, 109: 76-78.

9. Larsen WJ (2004) Human embryology. Churchill Livingstone, New York.

10. Natsis K, Konstantinos V, George T, Trifon T, Juergen $K$ (2010) A case of a supernumerary third head of biceps brachii muscle-clinical significance. Aristotle University Medical J, 37: 39-41.

11. Nayak SR, Ashwin Krishnamurthy, Latha V Prabhu, Jiji PJ, Lakshmi Ramanathan, Savinaya Kumar (2007) Multiple supernumerary muscles of the arm and its clinical significance. Bratisl Lek Listy, 109:74-76.

12. NayakSR, Ashwin K, Madhan KSJ, Latha VP, Vasudha S, Merin MT (2008) Four-headed biceps and triceps brachii muscles with neurovascular variation. Anat Sci Intl, 83: 107-111.

13. Ozan H, Atsev A, Sianau, A, Simsek, C, Basar R (1997) An unusual insertion of the accessory biceps brachii muscle. Am Anat Nippon, 72: 515-519.

14. Pacha VD, Forcada CP, Career BA, Liusa PM (2005) Innervation of biceps brachii and brachialis: anatomical and surgical approach. Clin Anatomy, 18: 186-194.

15. Paval J, Mathew JG (2006) A rare variation of the biceps brachii muscle. Indian J Plastic Surgery, 39: 65-67.

16. Poudal PP, Bhattarai C (2009) Study of the supernumerary heads of biceps brachii muscle in Nepalese. Nepal Med Coll J, 11: 96-98.

17. Rai R, Ranade AV, Prabhu LV, Prakash MMP (2007) Third head of biceps brachii: A study in indian population. Singapore Med J, 48: 929-931.

18. Rincon F, Rodriguez IZ, Sanchez A, Leon A, Gonzalez LF (2002) The anatomic characteristics of the third head of biceps brachii muscle in Colombian population. Rev Chis Anat, 20: 197-200.

19. Rodriguez-Niedenfuhr M, Vasquez T, Choi D, Parkin I, Sanudo JR (2003) Supernumerary humeral heads of biceps muscle revisited. Clin Anat, 16: 197-203.

20. Sandeep VP, Borole BS, Mahajan AA (2012) A study on the accessory head of biceps brachii in Indians which was done during cadaver dissections. J Clin Diagnostic Res, 6 (suppl.): 1137-1139.

21. Sargon MF, Tuncali D, Celik HH (1996) An unusual origin for the accessory head of biceps brachii muscle. Clin Anat, 9: 160-162.

22. Sharadkumar PS, Shaguphta TS, Rakhi MM (2012) Variant heads of biceps brachii muscle with clinical importance. Indian J Basic Applied Med Res, 1: 245-250.

23. Standring S (2005) Gray's anatomy. 39th Ed. In: Nervous system. Churchill Livingstone, Edinburgh, London, New York, Oxford, Philadelphia, Sydney, Toronto.

24. Vazquez T, Rodriguez-Niedenfuhr M, Parkin I, Sanudo JR (2003) A rare case of a four-headed biceps brachii muscle with a double piercing by the musculocutaneous nerve. Surg Radiol Anat, 25: 462-464.

25. Wood J (2012) Tables of primate head, neck, pectoral and upper limb muscles, anatomy and phylogeny of primate muscles and human evolution comparative. J Anat Physiol, 1: 44-59. 